\title{
Probing the Electrolyte Transfer in Ultrathin Polypyrrole Films by In Situ X-ray Reflectivity and Electrochemistry
}

\author{
Pirmin H. Lakner, Manuel Brinker, Christoph Seitz, Leon Jacobse, Vedran Vonk, Milena Lippmann,
} Sergey Volkov, Patrick Huber, and Thomas F. Keller*

Cite This: Langmuir 2020, 36, 13448-13456

Read Online

ABSTRACT: This study reports on the potential-induced charge and mass transfer between an ultrathin polypyrrole (PPy) film and an electrolyte by simultaneous in situ X-ray reflectivity (XRR) and electrochemistry (EC) utilizing their sensitivity to electrons. An about $30 \mathrm{~nm}$ thin PPy film was deposited on a silicon single crystal by fast potential cycling, providing a dense film of an extraordinary small surface roughness. XRR was recorded from the PPy film in an aqueous $0.1 \mathrm{M}$ perchloric acid at electric potentials between $-0.2 \mathrm{~V}$ and $+0.5 \mathrm{~V}$ vs $\mathrm{Ag} / \mathrm{AgCl}$. The PPy film shows typical reversible and linear changes in film thickness and electron density

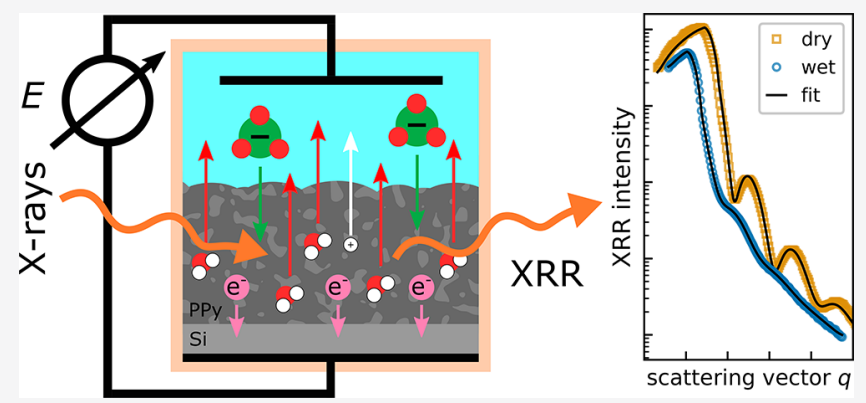
arising from the potential-dependent electrolyte incorporation. By introducing EC-XRR, a comprehensive analysis combining in situ XRR and EC, the net number of electrons passing through the PPy-electrolyte interface was deduced along with the potential-induced thickness variations, indicating a complex exchange mechanism. Evidently, along with the anion transfer, parallel charge compensation by protons and a volume and electron compensating counterflow of solvent molecules take place. Complementary time-dependent EC-XRR scans indicate that these exchange mechanisms are individual in two potential ranges. The low actuation along with a high pseudocapacitance suggest the fast potentiodynamically deposited PPy film as a promising supercapacitor material.

\section{INTRODUCTION}

Polypyrrole (PPy) is a highly attractive semiconducting polymer for various applications in the field of actuators, sensors, and (bio)microelectronics as, e.g., for $\mathrm{pH}$ sensors ${ }^{1}$ or drug release agents. ${ }^{2}$ It is also a promising electrode material for polymeric supercapacitors due to its high electrochemical (EC) pseudocapacitance, availability, environmental compatibility, and simple synthesis process. ${ }^{3-5}$ Additionally, PPy offers a further innovative aspect: Due to its high elasticity and stability, it enables the fabrication of flexible supercapacitors. ${ }^{4-6}$ The electrical conductivity of PPy in combination with its potential-induced ion incorporation and expulsion capabilities enable actuoric properties ${ }^{7}$ and thus permit the engineering of bioactuoric applications, like artificial muscles. ${ }^{8-10}$

An electric potential applied to a thin PPy film leads to the removal of electrons and to the generation of a charge imbalance, which is compensated by ion transfer between the film and the surrounding electrolyte, causing the PPy to swell. $^{11-15}$ The attainable magnitude of the potential-induced swelling is mostly determined by the type of synthesis ${ }^{16}$ and the composition of the electrolyte ${ }^{12-14}$ employed for the actuoric operation. The actuation for a given PPy film will be highest for sole ion incorporation, whereas mixed incorporation and excorporation of several species of the electrolyte solution lead to volume compensation. Both extreme cases are important, one for actuator applications, the other for supercapacitor optimization, indicating the need to identify and quantify the species involved. The amount and type of incorporated species are difficult to predict, partially since the interaction between PPy matrix, electrolyte, and solvent is manifold under an applied potential ${ }^{14,17}$ and partially, because such species cannot be easily discriminated or even quantified. Therefore, it is essential to determine the charge and material transport across the electrolyte-PPy interface, and the volume increase or swelling of the PPy film. While atomic force microscopy $(\mathrm{AFM})^{18,19}$ and bending experiments ${ }^{9,11,15}$ are mainly sensitive to changes in thickness and volume, and quartz crystal microbalance ( $\mathrm{QCM})^{20-22}$ is mainly sensitive to changes in mass, X-ray reflectivity (XRR) has the ability to simultaneously measure changes in thickness and electron density, ${ }^{23}$ opening a further perspective to observe the electrolyte transfer.

Received: July 14, 2020

Revised: October 19, 2020

Published: November 5, 2020 
This study elucidates the amount and type of species involved in the incorporation mechanism using EC-XRR, a novel approach relying on the combination of synchrotronbased in situ XRR and EC analysis. A $30 \mathrm{~nm}$ thin, dense, and smooth PPy film, deposited by fast potential-cycling, ${ }^{16,24}$ allows detailed XRR analysis while an electric potential is applied between $-0.2 \mathrm{~V}$ and $+0.5 \mathrm{~V}$ vs $\mathrm{Ag} / \mathrm{AgCl}$ in an aqueous electrolyte of $0.1 \mathrm{M}$ perchloric acid. EC-XRR permits one to simultaneously determine the swelling of ultrathin film systems, the electron transfer across the electrolyte-PPy interface and the transferred electric charge across the PPysubstrate interface. Overall, the here applied new approach of combining in situ XRR and EC analysis elucidates the electrical potential-dependent anion storage and the associated mechanisms of proton charge compensation and water displacement within ultrathin PPy films.

\section{EXPERIMENTAL SECTION}

All chemicals were used as received from Sigma-Aldrich, U.S.A. Pyrrole was distilled before use. Water was taken from an ultrapure water system (Veolia, Paris, France, $18.2 \mathrm{M} \Omega \mathrm{cm}$ ). All experiments were conducted at room temperature. All potentials in this study were measured and applied against a $\mathrm{Ag} / \mathrm{AgCl}$ reference electrode (Leakless Miniature $\mathrm{Ag} / \mathrm{AgCl}$ Reference Electrode, eDAQ PTY Ltd., Australia).

Sample Preparation and EC Cell. The sample substrate was a round (111)-oriented p-doped silicon wafer (Si-Mat, Kaufering, Germany) with $6 \mathrm{~mm}$ diameter and a height of $525 \mu \mathrm{m}$. Due to p-type doping with boron, the silicon wafer had a resistance of $0.01-0.02 \Omega \mathrm{cm}$. For an ohmic contact, a thin aluminum coating was applied on the backside of the silicon crystal. The silicon substrate was washed with isopropyl alcohol and acetone beforehand and stripped from native silicon oxide with ammonium fluoride $\left(\mathrm{NH}_{4} \mathrm{~F}\right.$, $40 \%$ in $\mathrm{H}_{2} \mathrm{O}$, Sigma-Aldrich, U.S.A.). $0.05 \mathrm{M}$ ammonium sulfite [ $\left(\mathrm{NH}_{4}\right)_{2} \mathrm{SO}_{3}$, Sigma-Aldrich, U.S.A.] was added to the ammonium fluoride solution to deoxygenate the solution to avoid etch pits in the silicon substrate caused by dissolved oxygen. ${ }^{25}$ The silicon substrate was immersed in this solution for $5 \mathrm{~min}$ and rinsed with deoxygenated $0.05 \mathrm{M}\left(\mathrm{NH}_{4}\right)_{2} \mathrm{SO}_{3}$ solution afterward and dried in a nitrogen flow. Directly after cleaning, the silicon crystal was placed in an electrochemical cell, electrically contacted from the back with a brass stamp and tightened at the sides with an O-ring made from fluororubber (FKM) to prevent electrolyte leakage. The EC cell permits a $4 \mathrm{~cm}$ high supernatant electrolyte over the sample. The reference electrode is entered inclined at half the height of the fluid column. A platinum wire coil with the same surface area as the silicon crystal was entered at the top of the liquid column as counter electrode. The EC cell is tightened with polytetrafluoroethylene (PTFE) screws and FKM O-rings to prevent loss of electrolyte. The EC cell is made from polyether ether ketone (PEEK), which provides high chemical stability and X-ray transparency. At the height of the crystal surface, the PEEK cell has a thin outer wall with a thickness of $0.5 \mathrm{~mm}$ to reduce residual absorption and scattering to a minimum. The active crystal surface is $0.221 \mathrm{~cm}^{2}$. Further details with a scheme and two photographs of the EC cell are provided in the Supporting Information, SI, Figure S1. The EC cell was built based on a modified design developed at the European Synchrotron Radiation Facility (ESRF) in Grenoble, France for X-ray analysis under electrochemical conditions. ${ }^{26}$ The EC cell was cleaned before experiments with diluted peroxymonosulfuric acid made with $1: 2: 9 \mathrm{v} / \mathrm{v} \mathrm{H}_{2} \mathrm{SO}_{4}(95-$ 98\%) $/ \mathrm{H}_{2} \mathrm{O}_{2}(30 \%) / \mathrm{H}_{2} \mathrm{O}$. A Metrohm Autolab PGSTAT204 potentiostat (Metrohm Autolab B.V., Utrecht, The Netherlands) was used to control the EC cell.

Polypyrrole Deposition. The PPy film was deposited electrochemically from a solution of $0.1 \mathrm{M}$ lithium perchlorate and $0.1 \mathrm{M}$ pyrrole in acetonitrile. A triangular potential waveform was applied for the cyclovoltammetric PPy film growth: The potential was swept between $-0.4 \mathrm{~V}$ and $+1.0 \mathrm{~V}$ for 200 cycles with a sweep rate $\nu$ of 1000
$\mathrm{mV} / \mathrm{s}$ beginning and ending at the open circuit potential (OCP) of about $+0.2 \mathrm{~V}$. The high sweep rate enabled the deposition of very smooth and adherent PPy films compared to galvanostatic deposition, potentiostatic deposition, and square wave deposition. ${ }^{24}$ The choice of $-0.4 \mathrm{~V}$ as lower limit ensured reduction of the PPy film and was still sufficiently anodic to avoid polymerization at the counter electrode. The upper limit of $+1.0 \mathrm{~V}$ permitted PPy deposition while avoiding PPy overoxidation. ${ }^{27}$ To mimic a linear sweep, the smallest potential step possible, $1 \mathrm{mV}$, was used to record both capacitive and faradaic effects. The current during the deposition of the PPy film and its oxidation/reduction was recorded with the potentiostat as a function of the applied potential. The resulting cyclic voltammogram $(\mathrm{CV})$ in Figure 1 shows a linear-periodic increase in current,

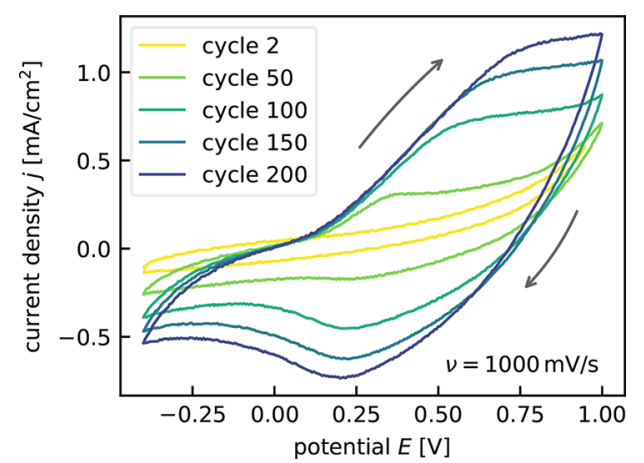

Figure 1. CV of the fast potentiodynamic PPy deposition. The potential was cycled 200 times between $-0.4 \mathrm{~V}$ and $+1.0 \mathrm{~V}$ with a potential sweep rate $\nu$ of $1000 \mathrm{mV} / \mathrm{s}$. The total deposition time was 560 s. The potential was applied against a $\mathrm{Ag} / \mathrm{AgCl}$ reference electrode.

representing the film growth. The electrochemical current is increasing for positive potential sweep as electric charge is consumed by pyrrole units for polymerization and deposition. ${ }^{28}$ As the number of cycles increases, stronger oxidation and reduction peaks become visible. Also, the absolute current increases as the film thickens and more material is reduced/oxidized, increasing the capacitance. ${ }^{24}$

After deposition, the sample was dismounted from the EC cell, washed with ultrapure water $(R \geq 18.2 \mathrm{M} \Omega)$, dried, and remounted in the EC cell. Dry XRR measurements were conducted and then, the sample was immersed in $0.1 \mathrm{M}$ aqueous perchloric acid $(\mathrm{pH}=1)$. Subsequently, the sample was cycled between $-0.2 \mathrm{~V}$ and $+0.5 \mathrm{~V}$ with $50 \mathrm{mV} / \mathrm{s}$ for five consecutive cycles to eliminate irreversible first cycle effects. ${ }^{29}$

X-ray Experiments. All X-ray experiments were conducted at beamline P08 at PETRA III at DESY, Hamburg, Germany. ${ }^{30}$ The used X-ray energy was $18 \mathrm{keV}(\lambda=0.069 \mathrm{~nm})$. The beam had a photon flux of about $10^{12}$ photons/second, a width of $1 \mathrm{~mm}$ and a height of $100 \mu \mathrm{m}$. A 6-Circle Kohzu NZD-3 diffractometer combined with a Dectris Pilatus $100 \mathrm{~K}$ area detector with two pairs of vertical and horizontal slits mounted on the detector arm was used to measure $\mathrm{X}$-ray reflectivity. Absorbers were used to reduce the incident beam intensity avoiding beam damage. All measurements were conducted on a horizontally aligned sample. All XRR data were analyzed with the software $\mathrm{GenX}^{31}$ using the Parratt-formalism. ${ }^{32}$ For a stable evaluation, XRR data sets were fitted with shared environmental parameters for instrument, substrate, residual silicon dioxide layer, and electrolyte, yielding the PPy film thickness, film roughness, and the electron density of the film.

\section{RESULTS AND DISCUSSION}

A perchlorate-doped PPy film was grown with electrodeposition by potential cycling on a silicon (111) single crystal as described above. The duration of the potentiodynamic deposition process was tuned to achieve a few $10 \mathrm{~nm}$ thick PPy film. The cyclic voltammogram in Figure 1 indicates 
a periodic, linear PPy film growth under the applied optimized parameters: The current originating from oxidizing and reducing the growing film increases linearly with applied cycles, and likewise the deposited charge increases linearly, being consumed by deposited PPy. This method of deposition with parallel oxidation/reduction leads to a smooth, adherent, and dense PPy film, as is typical for potentiodynamic PPy deposition. $^{16,24}$ First, the film was analyzed by X-ray reflectometry (XRR) in dry and wet conditions. Afterward, in aqueous electrolyte, XRR profiles were recorded at several constant potentials, while the transferred charges between the potential steps were derived from EC current integration. Combining XRR and EC data permits one to relate structural characteristics of the film like swelling induced thickness changes, electron density, and pseudocapacitance to the mechanism and type of molecular and ion transfer.

X-ray Reflectivity of the Dry and Wet Polypyrrole Film. Figure 2 shows the XRR profiles of the dry and wet Si-

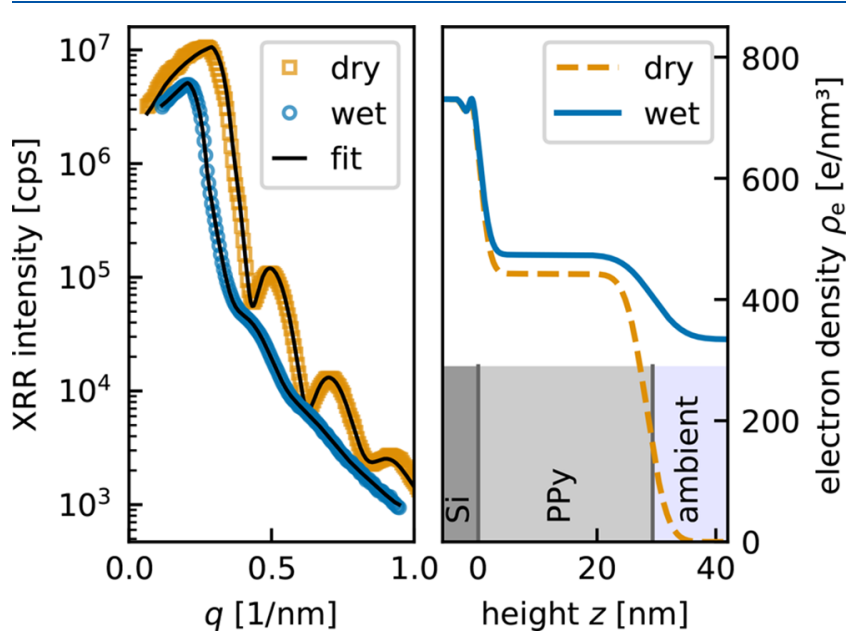

Figure 2. Fresnel-normalized XRR curves from the PPy film in ambient air and in electrolyte, respectively (left). The electron density profiles (right) are extracted from the XRR fits. The ambient medium is, depending on the measurement, air or electrolyte.

supported PPy film and the corresponding electron density profiles. XRR was recorded from the dry PPy film in ambient air after synthesis. Then, the film was immersed into electrolyte of aqueous $0.1 \mathrm{M} \mathrm{HClO}_{4}(\mathrm{pH}=1)$ and the measurement was repeated. Both measurements were conducted with open electric circuits, no potentials were applied. X-ray absorption systematically reduces the intensity of the XRR signal when ambient air is replaced by a liquid electrolyte. The amplitude of the oscillations decreased due to the decreased electron density contrast between the PPy film and environment, which also shifted the maximum of the XRR curve (i.e., the critical angle) to a lower scattering vector. Fitting a simulated curve to the XRR data using the Parratt formalism allows the film thickness and film density to be derived from the frequency and amplitudes of the oscillations of the XRR profiles in reciprocal space. $^{31-33}$

The results of the XRR fits listed in Table 1 show an increase in the thickness and the electron density of the film when immersed in the electrolyte solution. This is connected to the storage of the electrolyte and, in turn, induces a swelling of the dry PPy. The PPy film thickness increased from $28.3 \mathrm{~nm}$ in dry state by $3.7 \%$ to a thickness of $29.4 \mathrm{~nm}$. Swelling ratios from electrolyte wetting of about $11 \%$ and $70 \%$ were found for electrolyte infiltration in potentiostatically deposited PPy, ${ }^{18,34}$ which apparently has a higher porosity. The electron density increased by $7.1 \%$ due to wetting, i.e., incorporation of electrolyte and its solvent. The porosity of the PPy film can be estimated by comparing the electron densities of the dry and the wet PPy film by $\left(\rho_{\mathrm{e}, \mathrm{wet}}-\rho_{\mathrm{e}, \mathrm{dry}}\right) / \rho_{\mathrm{e}, \mathrm{H}_{2} \mathrm{O}}$. (The derivation of the formula is shown in the SI.) This reveals a porosity of (12 \pm 2 ) \%. The combination of potentiodynamic deposition with a high potential sweep rate and a high upper potential limit was deliberately chosen to achieve a high density and in turn a low porosity and low surface roughness ${ }^{24}$ as required for XRR experiments. Comparable studies show higher porosities for PPy films, but all were deposited statically (constant current or potential) or with lower potential sweep rates and potential limits. ${ }^{16,24,35-37}$ The real porosity of the PPy film is probably somewhat higher, as PPy films in ambient conditions contain condensed water from humidity. ${ }^{38}$ But even when this is taken into account, the porosity is remarkably low. The low porosity of the PPy film significantly increases the internal stability of the film and thus also explains the low wetting induced swelling. From XRR analysis a surface roughness of $2.7 \mathrm{~nm}$ was determined for the dry PPy film that changes to $4.0 \mathrm{~nm}$ after immersing the film into the electrolyte solution. The apparently low roughness of the dry PPy film is possibly due to water that accumulates on the typical cauliflower surface structure of PPy through condensation and capillary forces, making it appear smoother. This effect disappears when the electrolyte is added to the film and the XRR analysis shows the actual surface roughness of the PPy film. Future in situ attenuated total reflection Fourier transform infrared spectroscopy (ATR-FTIR) with polarization analysis ${ }^{39}$ may enlighten the existence of a preferred orientational molecular order of the PPy chains induced by the fast potential-cycling deposition method.

Electrochemical Film Response Observed by XRR. Subsequently, XRR scans were recorded while consecutively applying potentials of $-0.2 \mathrm{~V},+0.1 \mathrm{~V}$, and $+0.5 \mathrm{~V}$ for three cycles. The XRR profiles in Figure 3a show typical oscillations for thin film samples with a single layer. The curves were fitted to determine the thickness and the electron density profiles of the PPy film shown in Figure $3 \mathrm{~b}$. The fitted parameters are listed in Table 2 and plotted versus the applied potential in Figure 4, suggesting an electrochemically induced increase in PPy film thickness with increasing potential. The observed swelling indicates that the dominant mechanism is the incorporation of anions into the PPy film rather than the

Table 1. Results from XRR Fits of the PPy Film in Air and in Liquid Environment

PPy film parameters
thickness $(\mathrm{nm})$
electron density $\left(\mathrm{e} / \mathrm{nm}^{3}\right)$
roughness $(\mathrm{nm})$

PPy film parameters

electron density $\left(\mathrm{e} / \mathrm{nm}^{3}\right)$

roughness $(\mathrm{nm})$

$\begin{array}{rc}\text { in air } & \text { in electrolyte } \\ 28.3 & 29.4 \\ 442.1 & 473.2 \\ 2.7 & 4.0\end{array}$



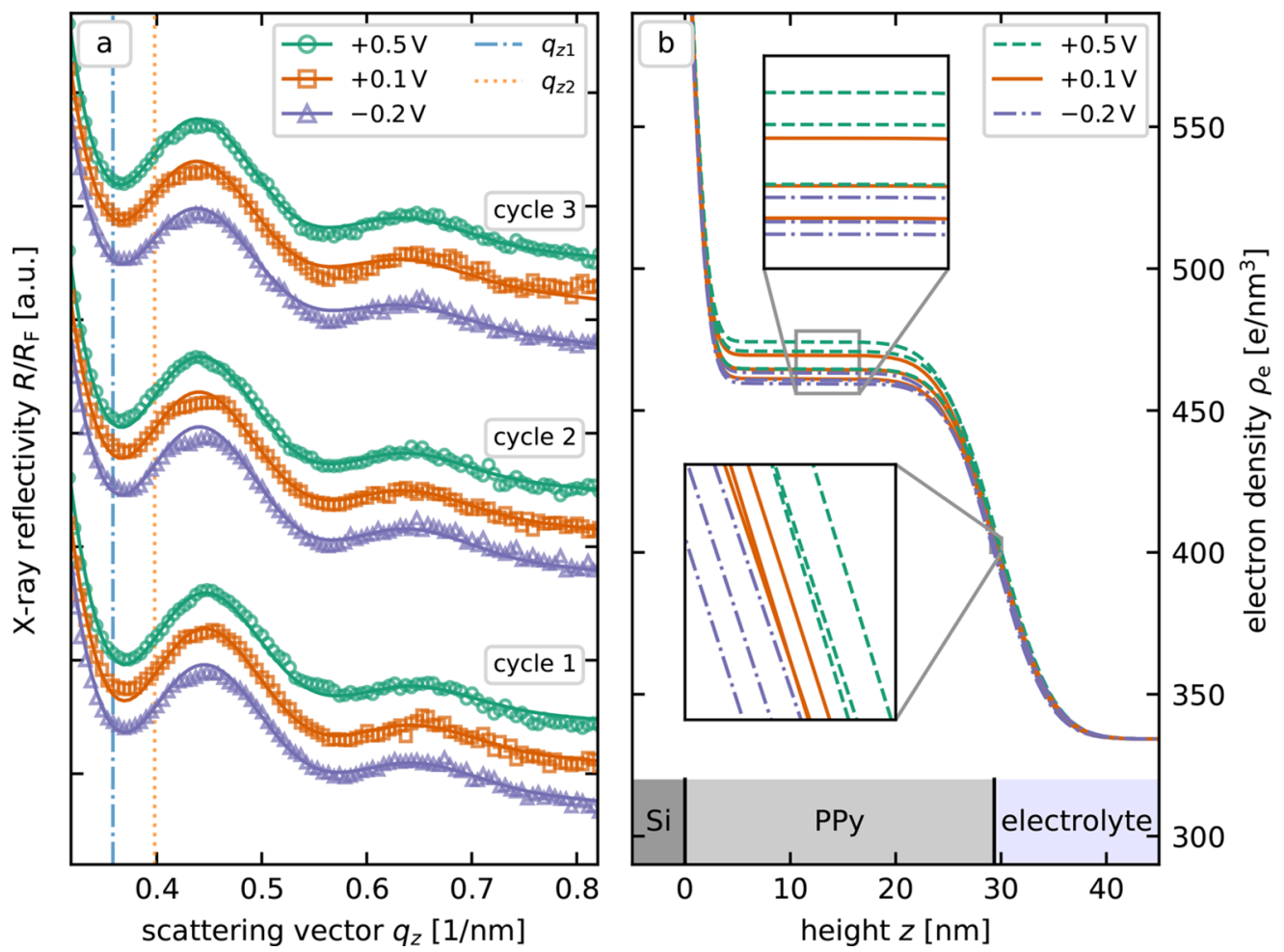

Figure 3. (a) Fresnel-normalized XRR curves and fits from the PPy film in electrolyte, measured at applied potentials of $-0.2 \mathrm{~V},+0.1 \mathrm{~V}$, and $+0.5 \mathrm{~V}$ over three cycles. The gray dashed and dotted lines represent the positions of the CV-XRR time scans. (b) The electron density profiles are extracted from the XRR fitting results. The insets are magnifications of the potential-dependent change in thickness and electron density. All potentials were applied against a $\mathrm{Ag} / \mathrm{AgCl}$ reference electrode.

Table 2. PPy Film Thickness, Electron Density, and Electron Transport via the Film Interfaces Derived from the XRR Analysis and the Current Integration

\begin{tabular}{|c|c|c|c|c|c|c|c|}
\hline \multirow[b]{2}{*}{ cycle } & \multirow[b]{2}{*}{$\begin{array}{c}\text { time } \\
(\mathrm{h}: \mathrm{mm})\end{array}$} & \multirow[b]{2}{*}{$E(\mathrm{~V})$} & \multirow[b]{2}{*}{$d(\mathrm{~nm})$} & \multirow[b]{2}{*}{$\begin{array}{c}\rho_{\mathrm{e}} \\
\left(\mathrm{e} / \mathrm{nm}^{3}\right)\end{array}$} & \multirow[b]{2}{*}{$\begin{array}{l}\text { electrons per area } \\
\left(\mathrm{e} / \mathrm{nm}^{2}\right)\end{array}$} & \multicolumn{2}{|c|}{ electron transfer from } \\
\hline & & & & & & $\begin{array}{l}\text { electrolyte to film } \\
\left(\mathrm{e} / \mathrm{nm}^{2}\right)(\text { XRR data })\end{array}$ & $\begin{array}{l}\text { film to substrate } \\
\left(\mathrm{e} / \mathrm{nm}^{2}\right)(\mathrm{EC} \text { data })\end{array}$ \\
\hline \multirow{4}{*}{1} & $0: 00$ & $\mathrm{OCP}$ & 29.36 & 473.2 & 13893 & 0.0 & 0.0 \\
\hline & $0: 15$ & 0.5 & 29.51 & 473.7 & 13981 & 88.0 & 7.6 \\
\hline & $0: 30$ & 0.1 & 29.49 & 469.0 & 13832 & -149.1 & -7.8 \\
\hline & $0: 44$ & -0.2 & 29.41 & 462.9 & 13615 & -217.0 & -13.0 \\
\hline \multirow[t]{3}{*}{2} & $0: 57$ & 0.5 & 29.83 & 470.4 & 14034 & 418.8 & 45.0 \\
\hline & $1: 15$ & 0.1 & 29.78 & 464.1 & 13819 & -214.3 & -16.5 \\
\hline & $1: 32$ & -0.2 & 29.70 & 459.1 & 13633 & -185.8 & -7.3 \\
\hline \multirow[t]{3}{*}{3} & $1: 45$ & 0.5 & 29.90 & 464.2 & 13880 & 246.9 & 38.3 \\
\hline & $1: 57$ & 0.1 & 29.82 & 460.7 & 13739 & -141.0 & -17.5 \\
\hline & $2: 20$ & -0.2 & 29.80 & 460.4 & 13717 & -21.9 & -11.6 \\
\hline
\end{tabular}

displacement of cations. ${ }^{40}$ The mean relative swelling per potential change yields a swelling ratio of $(0.54 \pm 0.07) \% / \mathrm{V}$, comparable, but somewhat smaller than the reported $0.9 \% / \mathrm{V}$ in aqueous $\mathrm{NaCl}$ solutions with a $\mathrm{pH}=1$, too. ${ }^{13}$ In a neutral aqueous $\mathrm{NaClO}_{4}$ solution, with no protons present that could compensate charges, the swelling capacity of PPy can be significantly higher. ${ }^{16}$

The swelling capacity of PPy films is described by the straincharge coefficient, which is obtained by normalizing the volume change to the transferred charge. For the here analyzed PPy film, a strain-charge coefficient of $(0.044 \pm 0.006) \mathrm{mm}^{3} / \mathrm{C}$ being within the range of reported PPy swelling capacities of $0.01 \mathrm{~mm}^{3} / \mathrm{C}$ and $0.51 \mathrm{~mm}^{3} / \mathrm{C}^{7,15}$ was derived from the potential-dependent thickness change, the area of the film of $0.221 \mathrm{~cm}^{2}$, and the transferred electric charge. Under the assumption of an exclusive and uninhibited transfer of perchlorate anions, and considering a molar volume of perchlorate dissolved in water of $49 \mathrm{~cm}^{3} / \mathrm{mol}^{41}$ a straincharge coefficient of $0.39 \mathrm{~mm}^{3} / \mathrm{C}$ can be calculated. Even higher strain-charge coefficients arise from additional effects such as accompanying hydration shells or osmotic diffusion ${ }^{42}$ that support further swelling. The here determined straincharge coefficient is only about $11 \%$ of the strain-charge coefficient of an exclusive perchlorate transfer. This small swelling capacity can be assigned to protons involved in the charge compensation, ${ }^{22,43}$ and an opposite solvent flow. ${ }^{21,44}$

From Figure $4 \mathrm{~b}$ it can further be noted that the electron density of the PPy film increases within the applied potential range from $-0.20 \mathrm{~V}$ to $+0.50 \mathrm{~V}$ in total by $(8.7 \pm 3.3) \mathrm{e} / \mathrm{nm}^{3}$, which corresponds to a relative increase of $(2.69 \pm 1.01) \% / \mathrm{V}$. 

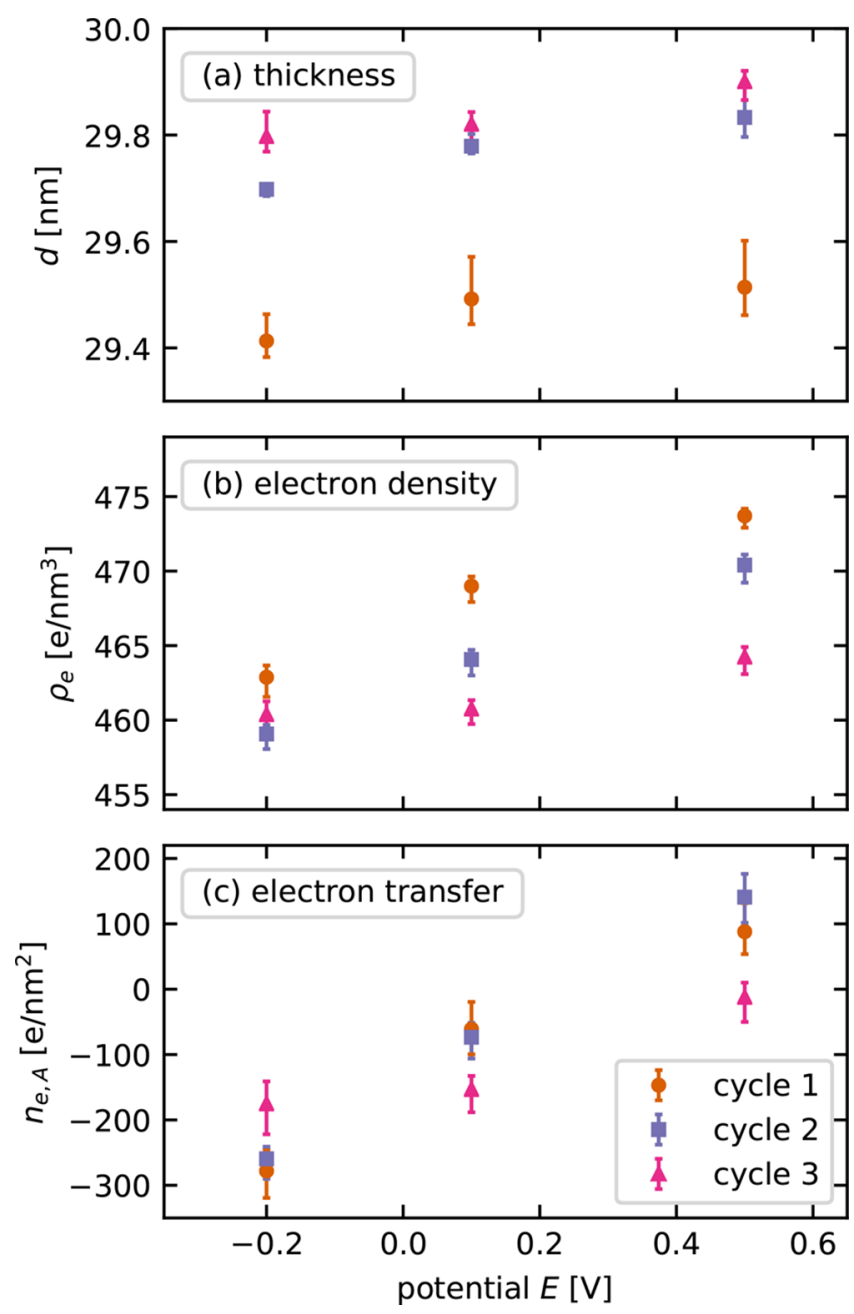

Figure 4. PPy film thickness (a), electron density (b), and electron transfer across the electrolyte-film interface (c) derived from the XRR fits as function of the applied potentials. All potentials were applied against a $\mathrm{Ag} / \mathrm{AgCl}$ reference electrode.

The relative change in electron density is five times higher than the relative change in film thickness, implying that the required space is compensated less by volume change than by material exchange, e.g., by a solvent counterflow providing volume for the anion influx.

The offset in thickness and electron density of the PPy film between the cycles in Figure $4 a, b$ can be explained by an overall potential-independent swelling of PPy by osmotic effects, ${ }^{42}$ causing the PPy film to become thicker and less dense per cycle. The concentrated electrolyte ions in the PPy matrix induce a water diffusion from the electrolyte into the PPy matrix to compensate for the concentration gradient. Potential cycling promotes this diffusion to converge toward a materialrelated limit and solely the potential-dependent swelling remains. ${ }^{13,14,16}$ However, the overall potential-dependence of the change in film thickness and density remains unaffected (see Figure 4a,b). The PPy film roughness was taken as one of the free fitting parameters and yielded an average value of $(4.04 \pm 0.06) \mathrm{nm}$. We did not observe any correlation to either the applied potential or the number of cycles (data not shown), and correspondingly omit here any further discussion.

Electrolyte Transfer as Function of the Applied Potential. The comparison of XRR scans at different potentials allows the determination of the number of electrons transferred across the film-electrolyte interface. The electron surface density,

$$
\rho_{A}=d \cdot \rho_{e}
$$

with $d$ and $\rho_{e}$ the film thickness and electron density, respectively, is the number of electrons buried under one film surface area unit. The difference between electron surface densities of two measurements with applied potentials $E_{1}$ and $E_{2}$ results in the area-normalized number of electrons transported over the electrolyte-film interface,

$$
n_{e, A}\left(E_{1} \rightarrow E_{2}\right)=\rho_{A, E_{2}}-\rho_{A, E_{1}}=d_{E_{2}} \cdot \rho_{e, E_{2}}-d_{E_{1}} \cdot \rho_{e, E_{1}}
$$

The resulting electron transfer listed in Table 2 is visualized in Figure 4c. It implies a linear increase in number of electrons transferred from the electrolyte to the PPy film with increased potential. A potential-dependent electron transfer of (441 \pm $67)$ e $/\left(\mathrm{nm}^{2} \mathrm{~V}\right)\left(R^{2}=0.86\right)$ is derived, which corresponds to a relative change in $\rho_{\mathrm{A}}$ of $(2.3 \pm 0.3) \%$ in the potential range used. The obvious linearity of the electron transfer suggests that the ability, quantity, and thus process of transferring electrons via the electrolyte-film interface remains stable for all three cycles even though the film shows irreversible changes in thickness and electron density.

The charge carrier transport taking place during the application of an electric potential is 2-fold at a PPy film: Electrons migrate across the PPy-substrate interface and, correspondingly, ions are crossing the electrolyte-PPy interface to balance the charge. XRR provides a measure to determine the electron density in the PPy film at different potentials, from which the number of electrons being part of the transferred ions and molecules, and, in turn, the electron transport via the electrolyte-PPy interface can be deduced. The electron transfer between film and substrate can be derived from the EC measurements recorded simultaneously to the XRR experiments. Integration of the recorded current after changing the applied potential supplies the transferred charge from the PPy film to the substrate. The resulting charge corresponds to the number of electrons that left the film to the substrate. To enable a comparison to the area-normalized number of electrons transported over the electrolyte-film interface determined by XRR, this charge was normalized to the geometrical electrode surface area. Dividing the numbers of transferred electrons derived from the XRR and EC measurements, listed in Table 2, gives the electron transfer ratio of the PPy film, i.e., the net number of electrons from the electrolyte incorporated into the PPy film per electron removed from the PPy film to the substrate. As Figure 5 shows, one electron crossing the film-substrate interface made $(8.9 \pm 1.2)$ electrons cross the electrolyte-film interface. For the assumption of a sole transfer of $\mathrm{ClO}_{4}^{-}$perchlorate anions, each containing 50 electrons, the electron transfer ratio should be 50 . However, the significantly lower value extracted is due to the contribution from protons to the charge exchange in the acidic solution (approximately $30 \%$ at $\mathrm{pH}=1^{22,43}$ ), and compensation of anion transfer by solvent movement, ${ }^{21,44}$ along the lines of the discussion above on the strain-charge coefficient.

Including the proton movement, the number of water molecules displaced from the PPy film per incorporated anion is about 2-3, which is in excellent agreement with former studies. ${ }^{21,44}$ This is a net change in film solvation and it cannot be excluded that the perchlorate anions move in the hydrated 


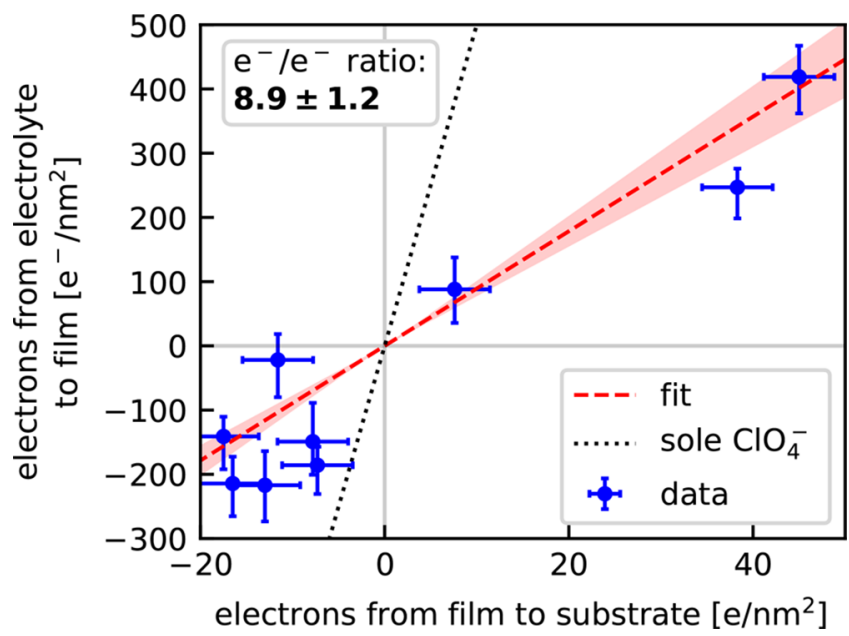

Figure 5. XRR deduced electron transport from electrolyte to PPy film as a function of EC-deduced electron transport from PPy film into the silicon substrate. The dashed linear fit reveals an electron transfer ratio of $8.9 \pm 1.2$. The dotted line shows the theoretical ratio for exclusive, uninhibited transport of perchlorate.

form as done in former studies. ${ }^{45,46}$ Both effects, proton transport and solvent displacement, also explain the low relative swelling capacity and the small strain-charge coefficient. These results could be supported by a follow-up in situ Rutherford backscattering spectroscopy (RBS) experiment, ${ }^{47}$ in which the change in elemental composition of the PPy film could be determined to confirm the proportion of transported anions.

The electrical impedance spectroscopy (EIS) data (see Figure S2) show two contributing effects within the investigated frequency range $(1 \mathrm{~Hz}$ to $100000 \mathrm{~Hz})$ : A constant fast capacitive contribution and a slower, potentialdependent contribution from the PPy film. The fast contribution originates from the electrochemical double layers formed at the interfaces. Their capacities are several orders of magnitude below the pseudocapacitance of the PPy layer and have therefore been neglected. The EIS data and the current integration data suggest another, much slower effect, possibly caused by a very slow Faradaic reaction. Due to this low speed, this effect was considered constant and accordingly subtracted in the current integration.

CV-XRR Time Scans. To monitor the transport across the electrolyte-film interface in response to faster potential cycling, a modified fast XRR measurement with increased time resolution was deployed while simultaneously applying a CV. For this purpose, the CV and XRR measurements had to be adjusted: The XRR intensity was determined at a fixed scattering vector, i.e., at fixed sample and detector angles to achieve a much higher temporal resolution of $5 \mathrm{~s}$. The scan rate of the $\mathrm{CV}$ was set to $1 \mathrm{mV} / \mathrm{s}$ to carefully change the potential per XRR signal acquisition, and to allow several cycles between $-0.2 \mathrm{~V}$ and $+0.5 \mathrm{~V}$. This was done consecutively at two different scattering vectors, at $0.36 \mathrm{~nm}^{-1}\left(q_{z 1}\right)$, a minimum, and at $0.40 \mathrm{~nm}^{-1}\left(q_{z 2}\right)$, an inflection point of the before recorded XRR oscillations. Correspondingly, the XRR intensities at these positions are sensitive to changes in the observed PPy film. The selected scattering vectors are shown as dashed lines in Figure 3a. The CV-XRR time scans in Figure $6 \mathrm{a} / \mathrm{b}$ clearly show that the XRR intensity decreases with an increasing applied potential. The change in XRR intensity is
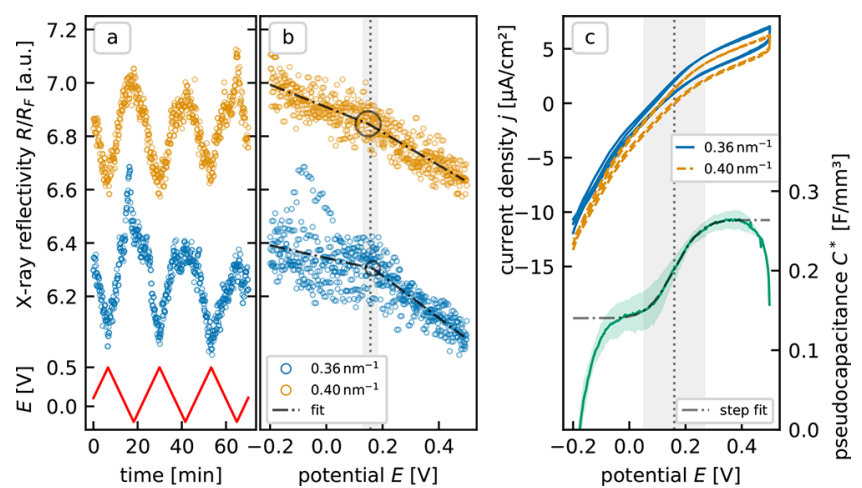

Figure 6. Time-resolved XRR signal at two scattering vectors while sweeping the applied potential between $-0.2 \mathrm{~V}$ to $+0.5 \mathrm{~V}$ with $1 \mathrm{mV} /$ s. XRR intensity and applied potential as function of time (a), XRR signal as function of the applied potential (b), and current density and from the latter derived pseudocapacitance as a function of the applied potential (c). All potentials were applied against a $\mathrm{Ag} / \mathrm{AgCl}$ reference electrode.

reproducible and reversible for both series over all cycles and the entire time, and is independent from the potential sweep direction. In the lower potential ranges, however, the XRR intensity seems to show a weaker change with potential. Fitting a piece-wise linear function with two ranges of different slopes results in a transition potential of $(0.157 \pm 0.028) \mathrm{V}$ (dashed line in Figure 6). Above this transition potential, the XRR signal reacts stronger to potential changes than below.

The $\mathrm{CV}$ recorded in parallel shows a similar behavior: In the higher potential range it shows a box-like, classic pseudocapacitive shape, while in the lower potential range the area of the $\mathrm{CV}$ is significantly smaller and the curve is steeper. From the differences in the current densities $\Delta j$ at the same potentials in a CV for up- and downward potential sweeps, the pseudocapacitance $C^{*}=\Delta j /(2 \nu d)$ can be calculated with the potential sweep rate $\nu$ and the film thickness $d^{48}$ The resulting pseudocapacitance shown in Figure 6c exhibits a typical step behavior in the central part of the applied potential range. ${ }^{40} \mathrm{~A}$ step function fitted to the data within the potential interval between $-0.05 \mathrm{~V}$ and $+0.40 \mathrm{~V}$ results in a transition potential of $0.160 \mathrm{~V}$ (dashed line) with a transition width of $0.108 \mathrm{~V}$ (light gray area). This transition potential is within the error in agreement with the one derived from the XRR data. The lower and higher plateau between $-0.10 \mathrm{~V}$ to $+0.10 \mathrm{~V}$ and $+0.20 \mathrm{~V}$ to $+0.4 \mathrm{~V}$ have a pseudocapacitance of $0.140 \mathrm{~F} / \mathrm{mm}^{3}$ and $0.264 \mathrm{~F} / \mathrm{mm}^{3}$, respectively. The latter value is $10 \%$ higher than recently reported for a potentiostatically deposited and otherwise similar PPy film. ${ }^{15}$ We assign the higher pseudocapacitance to arise from the lower porosity (a low volume ratio between pores and PPy) and the resulting higher mass density of the fast potentiodynamically deposited $\mathrm{PPy}$ film as compared to statically deposited PPy films. ${ }^{16,24,49} \mathrm{An}$ increased density of the PPy film does not necessarily lead to a higher internal surface, but it provides more PPy for charge storage and thus increases the volumetric pseudocapacitance of the film. By mass-normalization with the density of PPy of about $1.5 \mathrm{~g} / \mathrm{cm}^{3},{ }^{46}$ we estimate a pseudocapacitance of the PPy film of $176 \mathrm{~F} / \mathrm{g}$. This is in the range of the highest recently measured pseudocapacitances ${ }^{3,50}$ and as large as $28 \%$ of the theoretical maximum of the specific capacitance of PPy. ${ }^{51}$

It is evident from the independent transitions in the XRR signal and in the electrochemical CV that the PPy film changes 
its behavior at a potential of around $0.16 \mathrm{~V}$. The CV-XRR time scan measurement makes a qualitative statement about the process that has taken place: It clearly determines distinct mechanisms in the two regimes within the here investigated potential range. In the higher potential range, the PPy film shows a constantly high pseudocapacitance originating from the free anion intercalation process. In the lower potential range, the pseudocapacitance decreases to a lower, constant value. Here, a different and capacity-reducing process seems to take place, the PPy matrix becomes anion depleted and possibly proton transfer for charge compensation sets in. Anions dominate the exchange effect in the upper potential range and cations are likely contributing in the lower potential range. This is caused partly by electrostatic reasons, partly by the restricted resources given by the concentration in the pores. If anions cannot be expulsed from the PPy film, then ions from the opposite species, here protons, need to be incorporated. ${ }^{21,22,44,52}$ This is also supported by EIS (SI Figure S2) that shows a constant low conductivity below $0.2 \mathrm{~V}$ (SI Figure S4), caused by the absence of anions in the pores. Extended XRR experiments with a special design for improved time resolution ${ }^{53}$ could extent and substantiate the insight gained from this study.

\section{CONCLUSIONS}

This study presents the new methodology of EC-XRR, utilizing combined in situ XRR and EC analysis to quantify the electron transfer across liquid-solid electrolyte-PPy interfaces. As such, EC-XRR permits one to determine the potential-dependent properties of, e.g., ultrathin polymer films in wet environments relying on the intrinsic electron and electron transfer sensitivity of XRR and EC, respectively. For the here investigated ultrathin PPy film, EC-XRR elucidates the amount and type of species involved in the exchange mechanism as function of an applied electric potential. The EC-XRR deduced electron transfer ratio of electrons crossing the electrolyte-PPy and the PPy-Si interface indicates the displacement of 2-3 water molecules per incorporated perchlorate anion, assuming that protons are participating in charge compensation by about $30 \%$. Time-dependent EC-XRR scans further reveal distinct potential ranges representing two charge compensation processes, i.e., the conventional regime of dominant anion incorporation and the potential regime of anion depletion, where protons start to contribute to the charge transfer. Overall, EC-XRR shows that electrochemical deposition using fast potential cycling can be used to grow ultrathin, dense, and smooth PPy films. Their low actuation and high pseudocapacitance inferred from EC-XRR suggest the latter as promising candidates for supercapacitor electrode materials.

\section{ASSOCIATED CONTENT}

\section{SI Supporting Information}

The Supporting Information is available free of charge at https://pubs.acs.org/doi/10.1021/acs.langmuir.0c02068.

Details on the electrochemical cell used for the X-ray reflectivity experiment, the porosity calculations and the electrochemical impedance spectroscopy, and supplementary Figures S1-S4 (PDF)

\section{AUTHOR INFORMATION}

\section{Corresponding Author}

Thomas F. Keller - Deutsches Elektronen-Synchrotron DESY, Center for X-Ray and Nanoscience CXNS, Hamburg 22607, Germany; University of Hamburg, Department of Physics, Hamburg 20355, Germany; 이이.org/0000-0002-37706344; Email: thomas.keller@desy.de

\section{Authors}

Pirmin H. Lakner - Deutsches Elektronen-Synchrotron DESY, Center for X-Ray and Nanoscience CXNS, Hamburg 22607, Germany; University of Hamburg, Department of Physics, Hamburg 20355, Germany; 이이.org/0000-0002-24220433

Manuel Brinker - Hamburg University of Technology TUHH, Physics of Materials and High-Resolution X-Ray Analytics of the Structural Dynamics and Function of Matter, Hamburg 21073, Germany

Christoph Seitz - Deutsches Elektronen-Synchrotron DESY, Center for X-Ray and Nanoscience CXNS, Hamburg 22607, Germany; 이이이.org/0000-0002-8151-9933

Leon Jacobse - Deutsches Elektronen-Synchrotron DESY, Center for X-Ray and Nanoscience CXNS, Hamburg 22607, Germany; 이이이.org/0000-0002-2825-0963

Vedran Vonk - Deutsches Elektronen-Synchrotron DESY, Center for X-Ray and Nanoscience CXNS, Hamburg 22607,

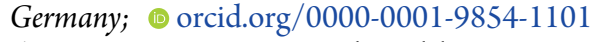

Milena Lippmann - Deutsches Elektronen-Synchrotron DESY, Hamburg 22607, Germany; ○ orcid.org/0000-0001-55986899

Sergey Volkov - Deutsches Elektronen-Synchrotron DESY, Hamburg 22607, Germany

Patrick Huber - Hamburg University of Technology TUHH, Physics of Materials and High-Resolution X-Ray Analytics of the Structural Dynamics and Function of Matter, Hamburg 21073, Germany; Deutsches Elektronen-Synchrotron DESY, Center for X-Ray and Nanoscience CXNS, Hamburg 22607, Germany; University of Hamburg, Center for Hybrid Nanostructures CHyN, Hamburg 22761, Germany; 이이이.org/0000-00022126-9100

Complete contact information is available at:

https://pubs.acs.org/10.1021/acs.langmuir.0c02068

\section{Notes}

The authors declare no competing financial interest.

\section{ACKNOWLEDGMENTS}

The authors gratefully acknowledge financial support from the German Research Foundation (DFG) within the Collaborative Research Initiative SFB 986 "Tailor-Made Multi-Scale Materials Systems-M3”, project number 192346071, subproject B7. The authors thank Florian Bertram for constructive discussions during the X-ray beamline experiment, Alexander Meinhardt for supporting AFM experiments, Abhisakh Sarma for assistance with the pyrrole distillation, and Markus Kowalski and his team for technical support (all DESY).

\section{REFERENCES}

(1) Carquigny, S.; Segut, O.; Lakard, B.; Lallemand, F.; Fievet, P. Effect of electrolyte solvent on the morphology of polypyrrole films: Application to the use of polypyrrole in $\mathrm{pH}$ sensors. Synth. Met. 2008, $158,453-461$. 
(2) Wadhwa, R.; Lagenaur, C. F.; Cui, X. T. Electrochemically controlled release of dexamethasone from conducting polymer polypyrrole coated electrode. J. Controlled Release 2006, 110, 531541.

(3) Bryan, A. M.; Santino, L. M.; Lu, Y.; Acharya, S.; D’Arcy, J. M. Conducting Polymers for Pseudocapacitive Energy Storage. Chem. Mater. 2016, 28, 5989-5998.

(4) Huang, Y.; Zhu, M.; Pei, Z.; Huang, Y.; Geng, H.; Zhi, C. Extremely Stable Polypyrrole Achieved via Molecular Ordering for Highly Flexible Supercapacitors. ACS Appl. Mater. Interfaces 2016, 8, 2435-2440.

(5) Huang, Y.; Li, H.; Wang, Z.; Zhu, M.; Pei, Z.; Xue, Q.; Huang, Y.; Zhi, C. Nanostructured Polypyrrole as a flexible electrode material of supercapacitor. Nano Energy 2016, 22, 422-438.

(6) Wang, Y.; Chen, F.; Liu, Z.; Tang, Z.; Yang, Q.; Zhao, Y.; Du, S.; Chen, Q.; Zhi, C. A Highly Elastic and Reversibly Stretchable AllPolymer Supercapacitor. Angew. Chem., Int. Ed. 2019, 58, 1570715711.

(7) Brinker, M.; Dittrich, G.; Richert, C.; Lakner, P.; Krekeler, T.; Keller, T. F.; Huber, N.; Huber, P. Giant electrochemical actuation in a nanoporous silicon-polypyrrole hybrid material. Science Advances 2020, 6, No. eaba1483.

(8) Valero, L.; Otero, T. F.; Martínez, J. G. Exchanged Cations and Water during Reactions in Polypyrrole Macroions from Artificial Muscles. ChemPhysChem 2014, 15, 293-301.

(9) Fuchiwaki, M.; Martinez, J. G.; Otero, T. F. Polypyrrole Asymmetric Bilayer Artificial Muscle: Driven Reactions, Cooperative Actuation, and Osmotic Effects. Adv. Funct. Mater. 2015, 25, 15351541.

(10) Zhang, B. G. X.; Spinks, G. M.; Gorkin, R.; Sangian, D.; Di Bella, C.; Quigley, A. F.; Kapsa, R. M. I.; Wallace, G. G.; Choong, P. F. M. In vivo biocompatibility of porous and non-porous polypyrrole based trilayered actuators. J. Mater. Sci.: Mater. Med. 2017, 28, 172.

(11) Pei, Q.; Inganas, O. Electrochemical applications of the bending beam method; a novel way to study ion transport in electroactive polymers. Solid State Ionics 1993, 60, 161-166.

(12) Gandhi, M.; Murray, P.; Spinks, G.; Wallace, G. Mechanism of electromechanical actuation in polypyrrole. Synth. Met. 1995, 73, 247-256.

(13) Kaneto, K.; Sonoda, Y.; Takashima, W. Direct Measurement and Mechanism of Electro-Chemomechanical Expansion and Contraction in Polypyrrole Films. Jpn. J. Appl. Phys. 2000, 39, 5918-5922.

(14) Takashima, W.; Pandey, S. S.; Fuchiwaki, M.; Kaneto, K. Cyclic Step-voltammetric Analysis of Cation-driven and Anion-driven Actuation in Polypyrrole Films. Jpn. J. Appl. Phys. 2002, 41, 75327536.

(15) Roschning, B.; Weissmüller, J. Stress-charge coupling coefficient for thin-film polypyrrole actuators - Investigation of capacitive ion exchange in the oxidized state. Electrochim. Acta 2019, $318,504-512$.

(16) Beebee, C.; Watkins, E. B.; Sapstead, R. M.; Ferreira, V. C.; Ryder, K. S.; Smith, E. L.; Hillman, A. R. Effect of electrochemical control function on the internal structure and composition of electrodeposited polypyrrole films: A neutron reflectometry study. Electrochim. Acta 2019, 295, 978-988.

(17) Zhu, J.; Xu, Y.; Wang, J.; Lin, J.; Sun, X.; Mao, S. The effect of various electrolyte cations on electrochemical performance of polypyrrole/RGO based supercapacitors. Phys. Chem. Chem. Phys. 2015, 17, 28666-28673.

(18) Smela, E.; Gadegaard, N. Volume change in polypyrrole studied by atomic force microscopy. J. Phys. Chem. B 2001, 105, 9395-9405.

(19) Higgins, M. J.; McGovern, S. T.; Wallace, G. G. Visualizing Dynamic Actuation of Ultrathin Polypyrrole Films. Langmuir 2009, $25,3627-3633$.

(20) Peres, R. C.; De Paoli, M.-A.; Torresi, R. M. The role of ion exchange in the redox processes of polypyrrole/dodecyl sulfate films as studied by electrogravimetry using a quartz crystal microbalance. Synth. Met. 1992, 48, 259-270.
(21) Vidanapathirana, K. P.; Careem, M. A.; Skaarup, S.; West, K. Ion movement in polypyrrole/dodecylbenzenesulphonate films in aqueous and non-aqueous electrolytes. Solid State Ionics 2002, 154$155,331-335$.

(22) Bose, C. S. C.; Basak, S.; Rajeshwar, K. Electrochemistry of poly(pyrrole chloride) films: a study of polymerization efficiency, ion transport during redox and doping level assay by electrochemical quartz crystal microgravimetry, $\mathrm{pH}$ and ion-selective electrode measurements. J. Phys. Chem. 1992, 96, 9899-9906.

(23) Steinrück, H.-G.; Will, J.; Magerl, A.; Ocko, B. M. Structure of n-Alkyltrichlorosilane Monolayers on $\mathrm{Si}(100) / \mathrm{SiO} 2$. Langmuir 2015, $31,11774-11780$.

(24) Otero, T. F.; De Larreta, E. Electrochemical control of the morphology, adherence, appearance and growth of polypyrrole films. Synth. Met. 1988, 26, 79-88.

(25) Fukidome, H.; Matsumura, M. Electrochemical study of atomically flattening process of silicon surface in $40 \%$ NH4F solution. Appl. Surf. Sci. 1998, 130-132, 146-150.

(26) Foresti, M. L.; et al. In situ X-ray analysis under controlled potential conditions: An innovative setup and its application to the investigation of ultrathin films electrodeposited on $\mathrm{Ag}(111)$. Electrochim. Acta 2006, 51, 5532-5539.

(27) Christensen, P.; Hamnett, A. In situ spectroscopic investigations of the growth, electrochemical cycling and overoxidation of polypyrrole in aqueous solution. Electrochim. Acta 1991, 36, 12631286.

(28) Sabouraud, G.; Sadki, S.; Brodie, N. The mechanisms of pyrrole electropolymerization. Chem. Soc. Rev. 2000, 29, 283-293.

(29) Li, Y. On the large overpotential of the first reduction of polypyrrole perchlorate films in organic solutions. Electrochim. Acta 1997, 42, 203-210.

(30) Seeck, O. H.; Deiter, C.; Pflaum, K.; Bertram, F.; Beerlink, A.; Franz, H.; Horbach, J.; Schulte-Schrepping, H.; Murphy, B. M.; Greve, M.; Magnussen, O. The high-resolution diffraction beamline P08 at PETRA III. J. Synchrotron Radiat. 2012, 19, 30-38.

(31) Björck, M.; Andersson, G. GenX: an extensible X-ray reflectivity refinement program utilizing differential evolution. $J$. Appl. Crystallogr. 2007, 40, 1174-1178.

(32) Parratt, L. G. Surface Studies of Solids by Total Reflection of XRays. Phys. Rev. 1954, 95, 359-369.

(33) Steinrück, H.-G. G.; Schiener, A.; Schindler, T.; Will, J.; Magerl, A.; Konovalov, O.; Li Destri, G.; Seeck, O. H.; Mezger, M.; Haddad, J.; Deutsch, M.; Checco, A.; Ocko, B. M. Nanoscale Structure of Si/ $\mathrm{SiO} 2 /$ Organics Interfaces. ACS Nano 2014, 8, 12676-12681.

(34) Pyo, M.; Kwak, C.-h. In situ scanning tunneling microscopy study on volume change of polypyrrole/poly(styrene sulfonate). Synth. Met. 2005, 150, 133-137.

(35) Hallik, A.; Alumaa, A.; Kurig, H.; Jänes, A.; Lust, E.; Tamm, J. On the porosity of polypyrrole films. Synth. Met. 2007, 157, 10851090.

(36) Ko, J. M.; Rhee, H. W.; Park, S.-M.; Kim, C. Y. Morphology and Electrochemical Properties of Polypyrrole Films Prepared in Aqueous and Nonaqueous Solvents. J. Electrochem. Soc. 1990, 137, 905.

(37) Satoh, M.; Kaneto, K.; Yoshino, K. Dependences of electrical and mechanical properties of conducting polypyrrole films on conditions of electrochemical polymerization in an aqueous medium. Synth. Met. 1986, 14, 289-296.

(38) Beck, F.; Oberst, M. Electrodeposition and cycling of polypyrrole. Makromol. Chem., Macromol. Symp. 1987, 8, 97-125.

(39) Keller, T. F.; Muller, M.; Ouyang, W.; Zhang, J.-T.; Jandt, K. D. Templating $\alpha$-Helical Poly(L-lysine)/Polyanion Complexes by Nanostructured Uniaxially Oriented Ultrathin Polyethylene Films. Langmuir 2010, 26, 18893-18901.

(40) Ren, X.; Pickup, P. G. Ion transport in polypyrrole and a polypyrrole/polyanion composite. J. Phys. Chem. 1993, 97, 53565362. 
(41) Conway, B. E.; Ayranci, E. Effective ionic radii and hydration volumes for evaluation of solution properties and ionic adsorption. $J$. Solution Chem. 1999, 28, 163-192.

(42) Bay, L.; Jacobsen, T.; Skaarup, S.; West, K. Mechanism of actuation in conducting polymers: Osmotic expansion. J. Phys. Chem. B 2001, 105, 8492-8497.

(43) Tsai, E. W.; Jang, G. W.; Rajeshwar, K. Proton transport accompanies redox switching of polypyrrole: A spectroelectrochemical study. J. Chem. Soc., Chem. Commun. 1987, 1776-1778.

(44) Pyo, M.; Reynolds, J. R. Potential dependent dual ion transport of conducting polymer: Redox polymer bilayers. J. Phys. Chem. 1995, 99, 8249-8254.

(45) López Cascales, J. J.; Fernández, A. J.; Otero, T. F. Characterization of the Reduced and Oxidized Polypyrrole/Water Interface: A Molecular Dynamics Simulation Study. J. Phys. Chem. B 2003, 107, 9339-9343.

(46) López Cascales, J. J.; Otero, T. F. Molecular dynamic simulation of the hydration and diffusion of chloride ions from bulk water to polypyrrole matrix. J. Chem. Phys. 2004, 120, 1951-1957.

(47) Khojasteh, N. B.; Apelt, S.; Bergmann, U.; Facsko, S.; Heller, R. Revealing the formation dynamics of the electric double layer by means of in - situ Rutherford backscattering spectrometry. Rev. Sci. Instrum. 2019, 90, No. 085107.

(48) Snook, G. A.; Kao, P.; Best, A. S. Conducting-polymer-based supercapacitor devices and electrodes. J. Power Sources 2011, 196, 112.

(49) Wang, J.; Xu, Y.; Wang, J.; Du, X.; Xiao, F.; Li, J. High charge/ discharge rate polypyrrole films prepared by pulse current polymerization. Synth. Met. 2010, 160, 1826-1831.

(50) Snook, G. A.; Chen, G. Z. The measurement of specific capacitances of conducting polymers using the quartz crystal microbalance. J. Electroanal. Chem. 2008, 612, 140-146.

(51) Lota, K.; Khomenko, V.; Frackowiak, E. Capacitance properties of poly(3,4-ethylenedioxythiophene)/carbon nanotubes composites. J. Phys. Chem. Solids 2004, 65, 295-301.

(52) Otero, T. F.; Martinez, J. G. Structural electrochemistry: Conductivities and ionic content from rising reduced Polypyrrole films. Adv. Funct. Mater. 2014, 24, 1259-1264.

(53) Lippmann, M.; Buffet, A.; Pflaum, K.; Ehnes, A.; Ciobanu, A.; Seeck, O. H. A new setup for high resolution fast X-ray reflectivity data acquisition. Rev. Sci. Instrum. 2016, 87, 113904. 